\title{
Caracterização dos ventos sobre a Flona Caxiuanã em ano de ENOS
}

\author{
Characterization of the winds over Flona Caxiuanã in the year of ENOS \\ Caracterización de los vientos sobre Flona Caxiuanã en el año de ENOS
}

Recebido: 31/10/2021 | Revisado: 08/11/2021 | Aceito: 10/01/2022| Publicado: 12/01/2022

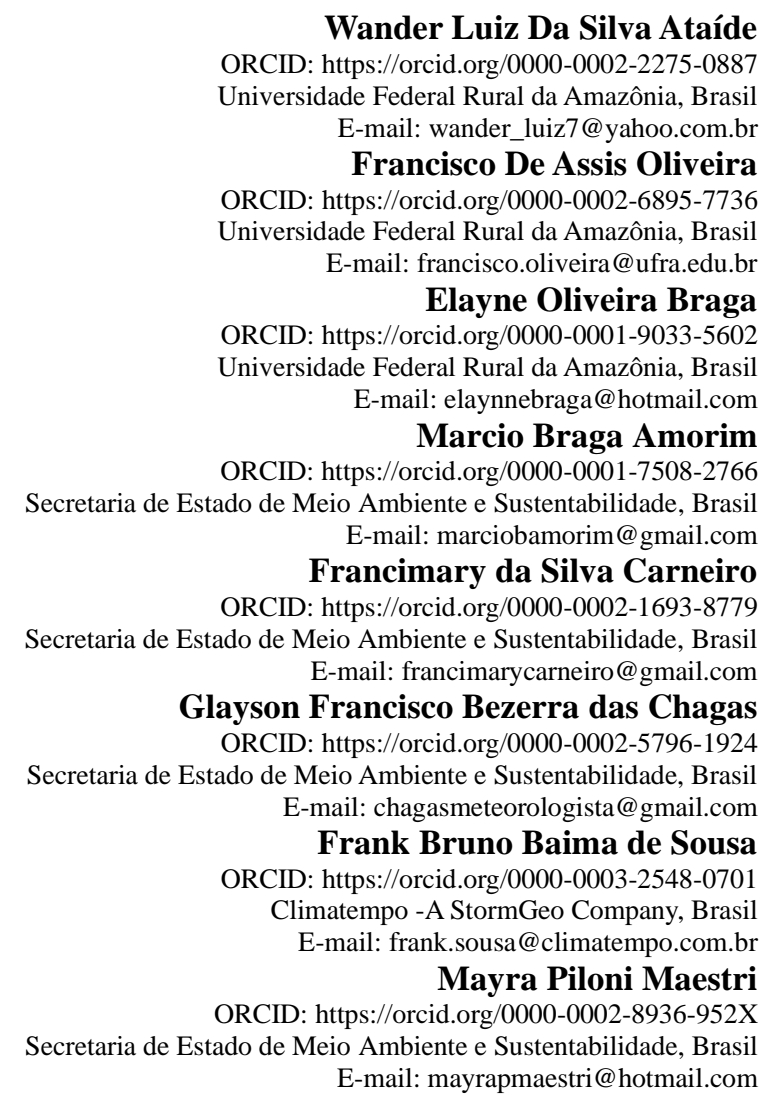

\section{Resumo}

Estudos sobre direção e velocidade dos ventos buscam retratar a distribuição espacial e temporal dos ventos nas regiões brasileiras, principalmente nas áreas de grande interesse socioambiental, como as florestas, em especial a floresta Amazônica. O objetivo do trabalho foi caracterizar o escoamento do vento como direção, velocidade e frequência do ar acima de um dossel a $56 \mathrm{~m}$ de altura. Um maior conhecimento das características de escoamento sobre a Flona Caxiuanã poderá levar à elaboração de melhores previsões de tempo locais, em relação à ocorrência e intensidade de precipitação, estimativa das temperaturas máximas e mínimas, alertas para ocorrência adversas à sociedade como ventos fortes, irrupção de incêndios e riscos no transporte fluvial. As médias anuais de velocidade foram 1,83 e 1,86 m.s-1, respectivamente. Quanto à classificação, nota-se claramente uma maior frequência em relação a ventos fracos para os anos de 2015 e 2016, com 54,57 e 56,50\% respectivamente. Medidas a 64 m de altura no km 67 na Flona Tapajós obtiveram um intervalo de classe variando de 2 a 3 m.s-1 com uma frequência de 52,7\%. Quanto à distribuição anual, nota-se claramente o predomínio dos ventos oriundos de NE e L (somados representam 45,45\% da captação dos ventos Alísios) para 2015, e de 65,97\% para 2016. Durante os anos de 2000 a 2004 , os valores mostraram-se iguais estatisticamente $\mathrm{p}>0,05$, com 1,60 e 1,77 m.s-1 para os períodos chuvoso e seco, respectivamente, e máximas de 1,79 m.s-1. A direção do vento predominantemente foi de NE e L durante os anos avaliados, estando em concordância com a maioria dos estudos situados na região de estudo em detrimento da confluência dos ventos Alísios, embora estando com sua intensidade reduzida em relação a anos ditos normais. A intensidade dos ventos não apresentou diferença estatística quando comparada por sazonalidade, apresentando poucas variações em relação à direção.

Palavras-chave: Direção; Velocidade; Ventos alísios. 


\begin{abstract}
Studies on wind direction and speed seek to portray the spatial and temporal distribution of winds in Brazilian regions, especially in areas of great socio-environmental interest, such as forests, especially the Amazon rainforest. The objective of the work was to characterize the wind flow like direction, speed and frequency of the air above a canopy at $56 \mathrm{~m}$ in height. Greater knowledge of the flow characteristics over the Flona Caxiuanã may lead to the elaboration of better local weather forecasts, in relation to the occurrence and intensity of precipitation, an estimate of maximum and minimum temperatures, alerts for adverse events to society such as strong winds, fires and risks in river transport. The annual speed averages were 1.83 and $1.86 \mathrm{~m} . \mathrm{s}-1$, respectively. As for the classification, there is clearly a higher frequency in relation to light winds for the years 2015 and 2016, with 54.57 and $56.50 \%$ respectively. Measured at 64 $\mathrm{m}$ in height at $\mathrm{km} 67$ in Flona Tapajós obtained a class interval ranging from 2 to $3 \mathrm{~m} . \mathrm{s}-1$ with a frequency of $52.7 \%$. As for the annual distribution, there is a clear predominance of winds from NE and L (together they represent $45.45 \%$ of the catchment of trade winds)for 2015, and $65.97 \%$ for 2016. During the years 2000 to 2004 , the values were statistically equal $\mathrm{p}>0.05$, with 1.60 and $1.77 \mathrm{~ms}-1$ for the rainy and dry periods, respectively, and maximum of 1.79 ms-1. The wind direction was predominantly NE and L during the years evaluated, in agreement with most studies located in the study region to the detriment of the confluence of the trade winds, although with its intensity reduced in relation to normal years. The intensity of the winds did not show statistical difference when compared by seasonality, presenting few variations in relation to the direction.
\end{abstract}

Keywords: Direction; Velocity; Trade winds.

\title{
Resumen
}

Los estudios sobre dirección y velocidad del viento buscan retratar la distribución espacial y temporal de los vientos en las regiones brasileñas, especialmente en áreas de gran interés socioambiental, como los bosques, especialmente la selva amazónica. El objetivo de este trabajo fue caracterizar el flujo del viento como dirección, velocidad y frecuencia del aire sobre un dosel de $56 \mathrm{~m}$ de altura. Un mayor conocimiento de las características del flujo sobre el Flona Caxiuanã podría conducir al desarrollo de mejores pronósticos meteorológicos locales, en relación a la ocurrencia e intensidad de precipitación, estimación de temperaturas máximas y mínimas, advertencias de eventos adversos a la sociedad como vientos fuertes, irrupciones de incendios y riesgos en el transporte fluvial. Los promedios anuales de velocidad fueron 1,83 y 1,86 m.s-1, respectivamente. En cuanto a la clasificación, se observa claramente una mayor frecuencia en relación a los vientos suaves para los años 2015 y 2016, con 54,57 y 56,50\% respectivamente. Medido a 64 m de altura en el km 67 en Flona Tapajós obtuvo un intervalo de clase que varió de 2 a 3 m.s- 1 con una frecuencia de $52,7 \%$. En cuanto a la distribución anual, hay un claro predominio de los vientos del NE y L (juntos representan el $45,45 \%$ de la captación de vientos alisios) para 2015, y el 65,97\% para 2016. Durante los años 2000 a 2004 , los valores fueron estadísticamente iguales p> 0.05, con 1.60 y $1.77 \mathrm{~ms}-1$ para los períodos lluvioso y seco, respectivamente, y un máximo de $1.79 \mathrm{~ms}-1$. La dirección del viento fue predominantemente NE y L durante los años evaluados, estando en concordancia con la mayoría de los estudios ubicados en la región de estudio en detrimento de la confluencia de los Alisios, aunque con su intensidad reducida en relación a los denominados años normales. La intensidad de los vientos no mostró diferencia estadística al compararla por estacionalidad, mostrando poca variación en relación a la dirección.

Palabras clave: Dirección; Velocidad; Vientos alisios.

\section{Introdução}

Caracterizar o escoamento do vento sobre obstáculos, especificamente a vegetação alta, não tem sido uma tarefa muito fácil para os cientistas, como no caso dos ecossistemas de floresta primária localizados nos trópicos, com média de 30 a $35 \mathrm{~m}$ de altura e algumas árvores atingindo facilmente $50 \mathrm{~m}$ de altura, dado o fato da mesma se revestir de grande complexidade temporal e espacial, em virtude das várias subcamadas que são originadas nas suas adjacências. Para isso necessitando de um aparato matemático e físico bastante robusto para caracterizar o movimento do vento.

O vento é o ar em movimento causado pelo aquecimento desigual da superfície terrestre, onde uma parcela de ar aquecida pelo sol fica menos densa e sobe, causando uma baixa pressão e deixando um espaço vazio no local, com isso, o ar frio adjacente tende a ocupar esse espaço (Abrão, 2005). Esse processo é gerado pela ação de gradientes de pressão atmosférica, sobre influência do movimento de rotação da Terra, da força centrífuga ao seu movimento e do atrito com a superfície, também sendo influenciado pelo contraste oceano-continental e topográfico (Varejão-Silva, 2006), fazendo com que a direção e intensidade sofram influências modificadoras ao longo de sua rota (Ayoade, 2007).

A velocidade do vento é dada pela diferença de pressão atmosférica, quanto maior a diferença de pressão em um determinado lugar, mais rápido será o escoamento (Mohmad, 2010). O vento possui grande importância ambiental, tendo em 
vista que, é responsável em grande parte pelas trocas de calor entre a vegetação e a atmosfera, além de atuar como facilitador do processo de transpiração das mesmas (Bueno et al., 2011).

A direção é de onde o vento emana, sendo geralmente descrita em graus azimute, ângulo formado entre o vetor vento e o norte geográfico (Varejão-Silva, 2006). A direção do vento tem influência sobre a dinâmica da convecção em determinadas regiões da Amazônia (Strong et al., 2005) e também serve para investigar o comportamento da circulação local e suas funções sobre o transporte de umidade (Santos et al., 2014). A direção é decorrente da posição do local em relação aos centros de pressão atmosférica, variando no espaço e no tempo, sofrendo influência da orografia e de obstáculos naturais e artificiais junto ao solo (Vendramini, 1986).

A interação do vento com as florestas causa mudanças no regime dos ventos locais, originando situações cujo estudo se manifesta de maneira complexo, dado o carácter turbulento e tridimensional do escoamento, na maior parte dos casos (Santos, 2013).

Estudos sobre direção e velocidade dos ventos possuem muita importância para a comunidade científica, e em alguns momentos escassos, não retratando a distribuição espacial e temporal dos ventos nas regiões brasileiras, principalmente nas áreas de grande interesse socioambiental, como as florestas, em especial as localizadas na Amazônica (Alves \& Silva, 2008).

Um maior conhecimento das características de escoamento sobre a Flona Caxiuanã poderá levar à elaboração de melhores previsões em relação à ocorrência e intensidade de precipitação, estimativa das temperaturas, alertas para ocorrência adversas à sociedade como ventos fortes, irrupção de incêndios e riscos no transporte fluvial e aéreo (Nogueira, 2008). Isso ocorre, sobretudo, em anos de eventos ENOS- El Niño Oscilação Sul, a qual desenvolveu-se a partir de setembro de 2015 e estendendo-se até abril de 2016, período este caracterizado como ENOS mais intenso já registrado.

Porém, estudar ecossistemas florestais representa um imenso desafio para a física do clima, sobretudo as florestas pristinas, a qual se reveste de grande complexidade acerca dos processos naturais que nela ocorrem. A determinação do campo de escoamento nas proximidades de uma árvore ou de um conjunto delas é fator importante nos estudos da engenharia de ventos (De Paula, 2007).

Um dos temas é o escoamento dos ventos em ecossistemas florestais, o qual é mais complexo em relação aos ecossistemas sem a presença de cobertura vegetal, isso por que, considera-se para os ecossistemas florestais o aquecimento/arrefecimento radiativo próximo ao solo e também os efeitos causados no dossel (Mahrt et al., 2000). Assim, o objetivo deste trabalho foi caracterizar a dinâmica de escoamento acima do dossel florestal durante o período chuvoso e seco utilizando dados de velocidade e direção do vento para os anos de 2015 e 2016, anos de ocorrência do El Niño. Com a hipótese de que o vento teria modificado sua direção e intensidade durante a ocorrência do ENOS.

\section{Metodologia}

A área experimental está situada na Flona Caxiuanã ( $01^{\circ} 42^{\prime} 30^{\prime \prime} \mathrm{S}, 51^{\circ} 31^{\prime} 45^{\prime}$ W; $60 \mathrm{~m}$ de altitude), distando cerca de $400 \mathrm{~km}$ a oeste da cidade de Belém, município de Melgaço (Souza Filho et al., 2005) (Figura 1). A região é caracterizada pela presença de floresta primária de terra firme, apresentando altura de dossel com aproximadamente 35 metros. 
Figura 1 - Localização do sítio na Floresta Nacional de Caxiuanã, Pará, Brasil e da Estação Científica Ferreira Pena.

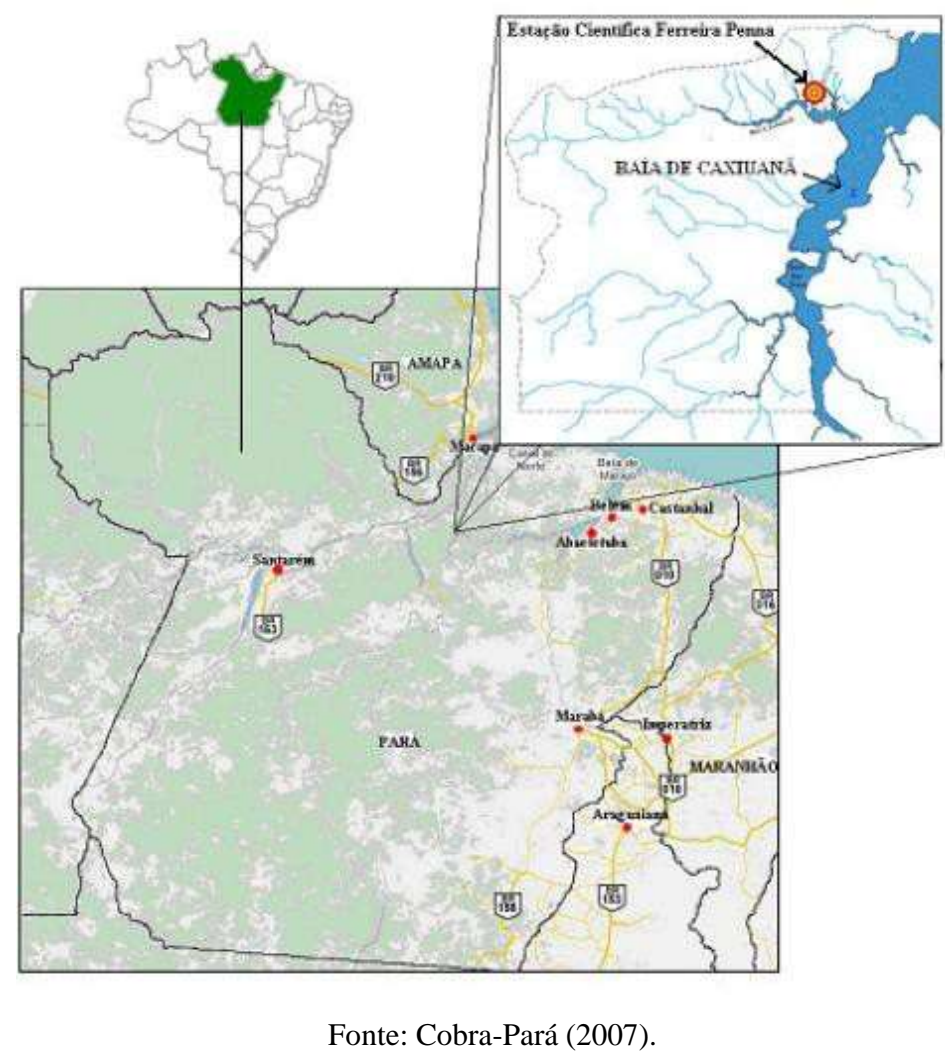

Foram realizadas médias da direção e velocidade do vento a cada meia hora, medidos por um anemômetro sônico 3D (CSAT-3D, Campbell Sci). A direção predominante foi caracterizada por meio de análise de frequência das observações para os anos compreendidos. A partir dos dados das componentes do vento foram obtidas a velocidade horizontal e a direção predominante. A série temporal foi analisada com base na classificação determinada a partir dos valores de máximo, mínimos e médios observados na região durante o tempo de observação, e foram classificados com base no estudo de Santos (2013), a qual categoriza os ventos em: forte, moderado e fraco. Os equipamentos foram instalados a $56 \mathrm{~m}$ de altura acima da superfície, pertencentes ao projeto Experimento de Grande Escala da Biosfera-Atmosfera (LBA).

A análise dos dados foi realizada com a utilização do Software Excel 2010 e os gráficos confeccionados com o software MatLab 2017 após remoção de outliers, seguindo instruções do fabricante. Foi realizado um teste de comparação de médias para confrontar as velocidades do vento sazonalmente e entre os anos compreendidos, para tal foi utilizado o Software Minitab®4.

\section{Resultados e Discussão}

A média anual da direção apresentou ventos predominantemente de nordeste, para os anos de 2015 e 2016 (Figura 2). E para a velocidade, as médias anuais foram 1,83 e 1,86 m.s-1, respectivamente. Este padrão corrobora com a maioria dos estudos, em decorrência do sítio experimental situar-se na zona de confluência dos ventos Alísios. As máximas obtidas para os respectivos anos foram 6,31 e 8,05 m.s-1. A provável explicação para a aproximação das médias anuais talvez se dê pelo fato de os dois anos avaliados ainda apresentarem os efeitos do El Niño (o segundo semestre de 2015 e parte do primeiro semestre de 2016) fazendo com que as velocidades médias ficassem próximas umas das outras. 
Figura 2 - Médias e máximas da velocidade e direção do vento (A) 2015 e (B) 2016 na Floresta Nacional de Caxiuanã, Pará, Brasil.
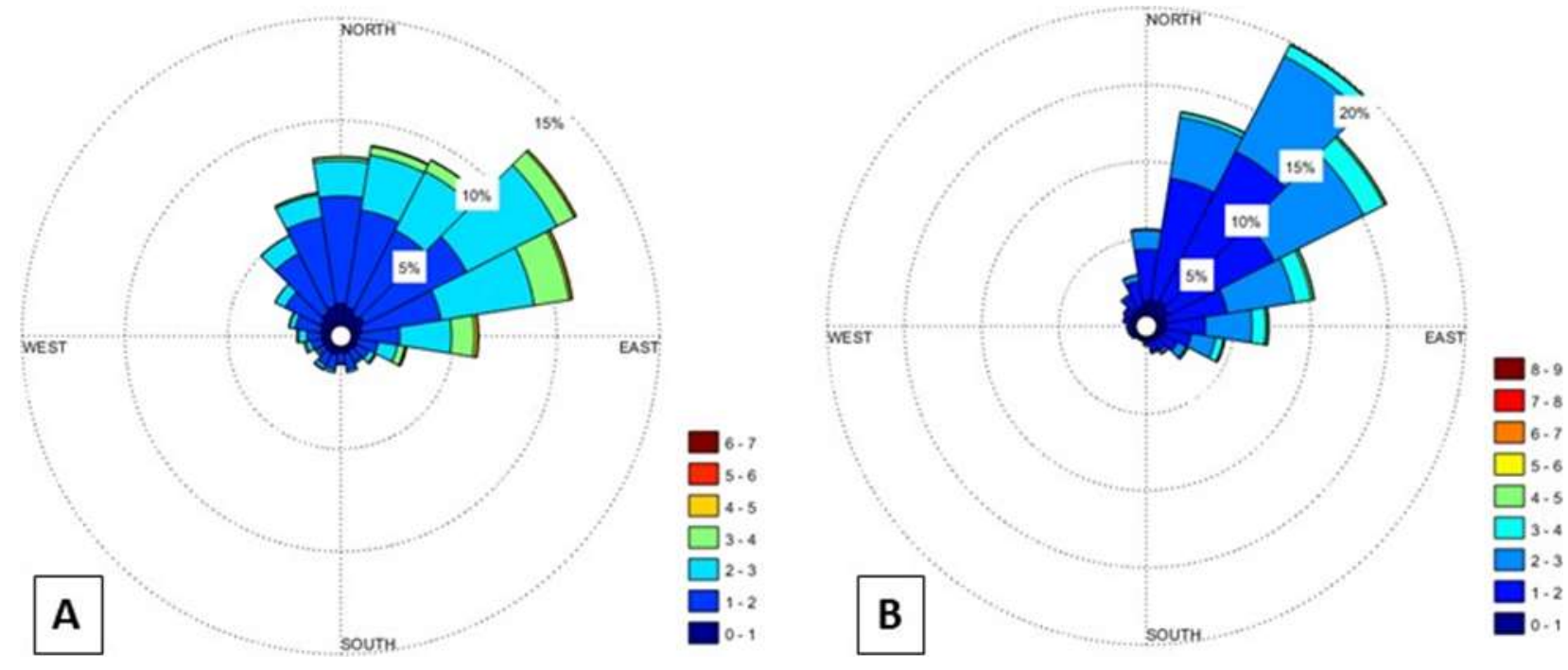

Fonte: Autores.

Oliveira e Fisch (2001) obtiveram máximas de 9,6 m.s ${ }^{-1}$ em uma floresta intacta (Ji Paraná - Rondônia), com médias de $2 \mathrm{~m} . \mathrm{s}^{-1}$ a mais do que em áreas de pastagem à $80 \mathrm{~km}$ de distância da área de estudo, onde a velocidade do vento acima do dossel começa perder intensidade a partir das 13h. Já nesse mesmo sítio, Nunes et al (2010) obtiveram médias de 1,69 e máximas de 1,79 m.s ${ }^{-1}$ para o ano de 2004, que foiconsiderado ano de El Niño de fraca intensidade.

Na região Tropical, tem sido constatada uma alternância na componente zonal (Leste-Oeste) do vento, a qual inverte a direção a cada intervalo de aproximadamente 26 meses (Hastenhrath, 1985). Em um fenômeno conhecido como oscilação quase bianual do vento (Varejão-Silva, 2006).

Quando separados por sazonalidade, os dois anos avaliados também demonstraram que a velocidade do vento foi maior no período seco de 2016 (Tabela 1), talvez até pela perda de intensidade da metade para o final do primeiro semestre de 2016, condizendo com a menor cobertura de nuvens e enfraquecimento do fenômeno, a qual proporciona uma maior velocidade horizontal do vento. Onde também o enfraquecimento e a direção do vento predominantemente de NE-L, correspondem à zona de confluência dos ventos Alísios.

Tabela 1 - V.V velocidade média diária do vento $\left(\mathrm{m} . \mathrm{s}^{-1}\right)$ e D.V direção do vento $\left(^{\circ}\right)$ para os períodos diurno e noturno em 2015 e 2016 na Floresta Nacional de Caxiuanã, Pará, Brasil. Em que: V.V = Velocidade do vento (m.s $\left.{ }^{-1}\right)$ e D.V = direção do vento $\left({ }^{\circ}\right)$.

\begin{tabular}{|c|c|c|c|c|c|c|c|c|c|}
\hline \multicolumn{5}{|c|}{2015} & \multicolumn{5}{|c|}{2016} \\
\hline & \multicolumn{2}{|c|}{ Chuvoso } & \multicolumn{2}{|c|}{ Seco } & & \multicolumn{2}{|c|}{ Chuvoso } & \multicolumn{2}{|c|}{ Seco } \\
\hline & V.V & D.V & V.V & D.V & & V.V & D.V & V.V & D.V \\
\hline Diurno & 1.94 & 139.05 & 1.92 & 130.97 & Diurno & 1.94 & 99.27 & 1.98 & 79.76 \\
\hline Noturno & 1.59 & 152.97 & 1.87 & 126.59 & Noturno & 1.68 & 95.08 & 1.83 & 71.79 \\
\hline
\end{tabular}

Fonte: Autores.

Para esta mesma tabela anterior (Tabela 1), foi realizado o teste de comparação de médias (Tabela 2) entre os anos e períodos sazonais compreendidos. Do qual não foi possível detectar diferença estatísticas entre os mesmos. 
Tabela 2 - Análise estatística para comparação de médias da velocidade do vento para os anos de 2015 e 2016 na Flona Caxiuanã, Pará, Brasil.

\begin{tabular}{|c|c|c|c|c|c|}
\hline Source & DF & SS & MS & $\mathrm{F}$ & $p$ \\
\hline Anos & 1 & 0.177 & 0.177 & 0.22 & 0.642 \\
\hline Error & 818 & 670.148 & 0.819 & & \\
\hline Total & 819 & 670.325 & & & \\
\hline S: & 0.9051 & R-Sq: & $0.03 \%$ & $\mathrm{R}-\mathrm{Sq}(\operatorname{adj})$ & $0.00 \%$ \\
\hline \multicolumn{6}{|c|}{ Individual 95\% CIs For Mean Based on } \\
\hline Nível & $\mathrm{N}$ & Mean & Std. Dev & & \\
\hline 1 & 410 & 1.9098 & 0.933 & & \\
\hline 2 & 410 & 1.8805 & 0.8764 & & \\
\hline \multicolumn{6}{|c|}{ Grouping Information Using Tukey Method } \\
\hline Anos & $\mathrm{N}$ & Mean & Grouping & & \\
\hline 1 & 410 & 1.9098 & A & & \\
\hline 2 & 410 & 1.8805 & A & & \\
\hline
\end{tabular}

Fonte: Autores.

Em estudo realizado por Cohen et al. (2006) na Flona Tapajós foram diagnosticado a canalização dos ventos devido a presença da enseada, mas nem tanto aos efeitos topográficos. Isto é valido para este estudo tendo em vista que Caxiuanã não dispõe de terrenos complexos.

As Flonas de Caxiuanã e Tapajós apresentam confluência dos ventos alísios, e também brisa oriunda de suas enseadas, devido a este fato também fez-se a classificação da direção em quatro grandes setores: Norte (N) Sul (S), Leste (L) e Oeste (O) e em análise mais detalhada em subsetores: Nordeste (NE), Sudeste (SE), Noroeste (NO) e Sudoeste (SO), esta última posição fez referência à localização da enseada de Caxiuanã em relação à torre micrometeorológica.

Eventos noturnos de rajadas de vento foram registrados em Caxiuanã durante a estação seca (Nogueira, 2008). A calmaria antecedente à ocorrência das rajadas pode estar associada à circulação local que se estabelece pouco antes do pôr-dosol, em que devido ao resfriamento radiativo, o ar acima da floresta se torna estável, enquanto que, o ar acima da baía de Caxiuanã é instável, em função das elevadas temperaturas de suas águas (André; Mahrt, 1982). Esta circulação entre a floresta e a baía geraria correntes descendentes de ar sobre a floresta, caracterizando calmaria, o que de fato pôde ser observado através da ocorrência de ventos fracos durante o período noturno.

A gênese das rajadas de vento observadas em Caxiuanã pode estar associada à existência de uma enseada à sudoeste (SO) do ponto de mediação e à atividade convectiva local, a qual promovem a convergência do escoamento noturno e a formação de intensas correntes de ar descendentes sobre a floresta (Nogueira, 2008).

A Organização Mundial de Meteorologia - WMO recomenda como horários ideais para monitoramento das principais observações meteorológicas (9, 12 e 18 horas), utilizou-se neste estudo os horários de 9, 12 e 15 horas (horário local) pelo fato de ser o período do dia em que os ventos apresentam maior intensidade, como demonstrado pela Figura 29 (A e B - 9h no período chuvoso para 2015 e 2016, respectivamente; C e D - 12h; E e F - 15h) e Figura 30 (A e B - 9h seco 2015 e 2016; C e D - 12h; E e F - 15h).

A velocidade às 9h (Tabela 3) durante o período chuvoso foi superior em 2015 em relação ao ano de 2016. Isso talvez possa ser explicado pela atipicidade relacionado ao primeiro semestre de 2015, com médias anuais aumentando e não reduzidas como é de se esperar para um ano de ENOS, às 12h 2016 > 2015 e às 15h 2015 > 2016. Às 9h durante o período seco de $2015<2016$, às 12 h de $2015<2016$ e às 15 h de $2015<2016$. 
Tabela 3. Distribuição da velocidade e direção do vento sobre a Flona Caxiuanã durante o evento de ENOS 2015-2016.

\begin{tabular}{|c|c|c|c|c|c|}
\hline & & \multicolumn{2}{|c|}{ Velocidade } & \multicolumn{2}{|c|}{ Direção } \\
\hline & & 2015 & 2016 & 2015 & 2016 \\
\hline \multirow[t]{2}{*}{$9 \mathrm{~h}$} & Chuvoso & $1.85{\mathrm{~m} . \mathrm{s}^{-1}}^{-1}$ & $1.73{\mathrm{~m} . \mathrm{s}^{-1}}^{-1}$ & $117.26^{\circ}(\mathrm{SE})$ & $54.28^{\circ}(\mathrm{NE})$ \\
\hline & Seco & $2.25 \mathrm{~m} \cdot \mathrm{s}^{-1}$ & $2.45 \mathrm{~m} \cdot \mathrm{s}^{-1}$ & $150.68^{\circ}(\mathrm{SE})$ & $82.52^{\circ}(\mathrm{L})$ \\
\hline \multirow[t]{2}{*}{$12 \mathrm{~h}$} & Chuvoso & $2.24{\mathrm{~m} . \mathrm{s}^{-1}}^{-1}$ & $2.16 \mathrm{~m} . \mathrm{s}^{-1}$ & $135.56^{\circ}(\mathrm{SE})$ & $73.78^{\circ}(\mathrm{NE})$ \\
\hline & Seco & $2.08 \mathrm{~m} \cdot \mathrm{s}^{-1}$ & $2.13 \mathrm{~m} \cdot \mathrm{s}^{-1}$ & $122.63^{\circ}(\mathrm{L})$ & $85.66^{\circ}(\mathrm{L})$ \\
\hline \multirow[t]{2}{*}{$15 \mathrm{~h}$} & Chuvoso & $2.23 \mathrm{~m} \cdot \mathrm{s}^{-1}$ & $1.98 \mathrm{~m} \cdot \mathrm{s}^{-1}$ & $132.07^{\circ}(\mathrm{SE})$ & $96.21^{\circ}(\mathrm{L})$ \\
\hline & Seco & $1.95 \mathrm{~m}^{\mathrm{s}} \mathrm{s}^{-1}$ & $2.01 \mathrm{~m} . \mathrm{s}^{-1}$ & $125.91^{\circ}(\mathrm{SE})$ & $109.5^{\circ}(\mathrm{SE})$ \\
\hline
\end{tabular}

Fonte: Autores.

Durante o período chuvoso a direção predominante do vento para os mesmos horários (Tabela 5) foram: às 9h de 2015 $\left(150,68^{\circ}\right) \mathrm{SE}$ e $2016\left(82,52^{\circ}\right) \mathrm{L}$, às $12 \mathrm{~h} 2015\left(122,63^{\circ}\right) \mathrm{L}$ e $2016\left(85,66^{\circ}\right) \mathrm{L}$, e às $15 \mathrm{~h} 2015\left(125,91^{\circ}\right) \mathrm{SE}$ e $2016\left(109,50^{\circ}\right) \mathrm{SE}$. Para o período seco, às 9h $2015\left(117,26^{\circ}\right)$ SE e $2016\left(54,28^{\circ}\right)$ NE, às 12h $2015\left(135,56^{\circ}\right)$ SE e $2016\left(73,78^{\circ}\right)$ NE, e às 15h $2015\left(132,07^{\circ}\right) \mathrm{SE}$ e $2016\left(96,21^{\circ}\right) \mathrm{L}$.

Pôde-se comprovar a menor intensificação dos ventos para o ano de 2015 (El Niño de forte intensidade) em comparação ao ano de 2016. Alguns trabalhos como de Molion (2017); Melo et al (2015); Barnard et al (2016); Silva e Salvador (2016) apontaram de fato que os anos de 2015 e 2016 registraram um evento ENOS de forte intensidade. 
Figura 3 - Distribuição das direções e horários predominantes do vento para os período chuvoso de 2015 e 2016 às 9h A e B, 12h C e D, 15h E e F na Floresta Nacional de Caxiuanã,Pará, Brasil.
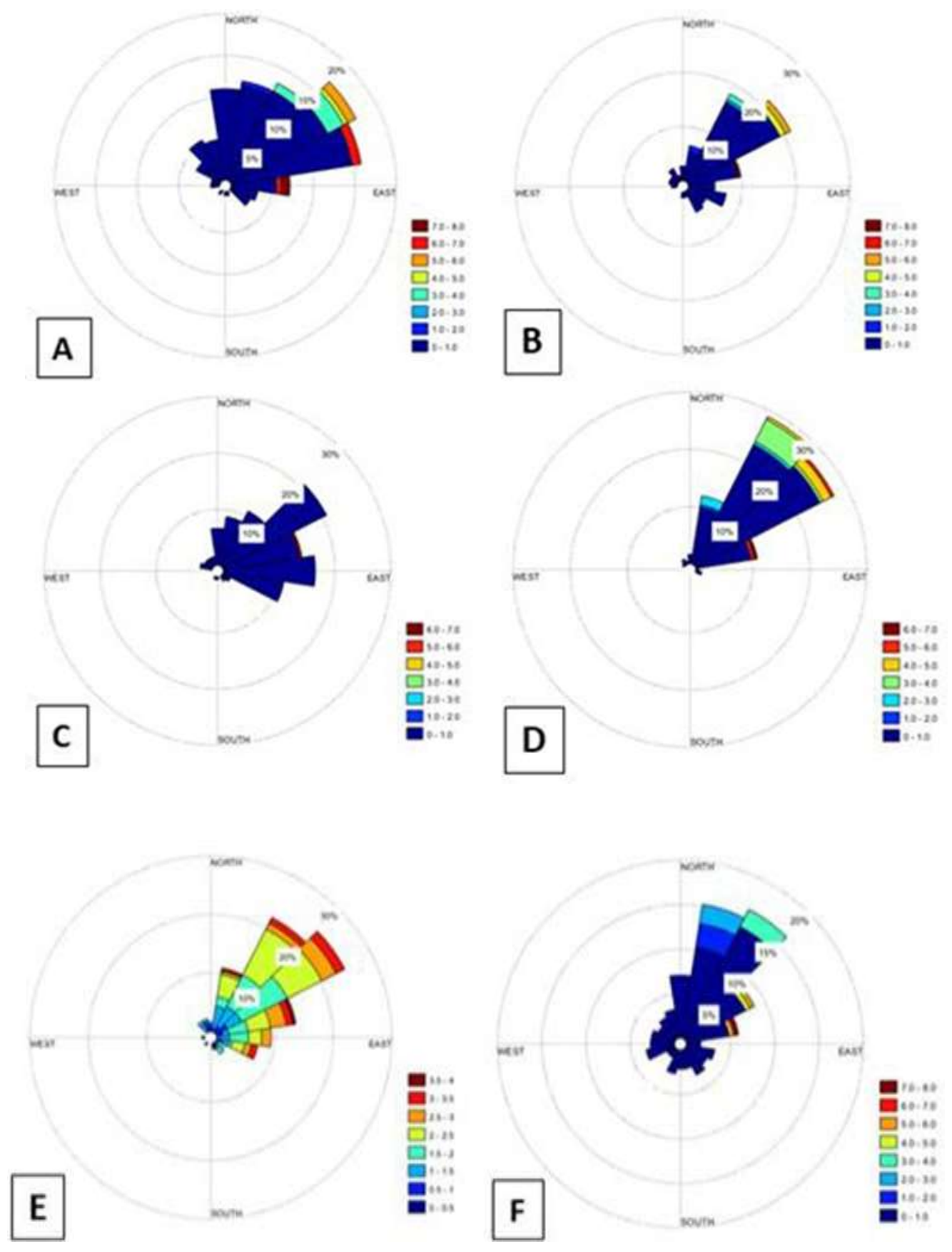

Fonte: Autores.

A maioria das frequências mudam entre os setores NE (Figura 4) e SE, o que pode estar relacionado a variabilidade sazonal dos ventos Alísios (Santos et al. 2014). Onde na estação chuvosa de um ano normal o padrão de circulação de grande escala intensifica os ventos Alísios de NE sobre a região Amazônica, devido à alta subtropical do Atlântico Norte, a qual é mais forte do que a alta subtropical do Atlântico Sul. Já o padrão oposto incrementa os ventos Alísios de SE, o qual ocorre na estação menos chuvosa. Na estação chuvosa os ventos são mais frequentes ao setor NE, enquanto que, na estação menos chuvosa são mais frequentes ao setor SE (Santos et al. 2014). Em anos normais há pouca variação entre as direções SE e NE, tanto na estação chuvosa quanto seca, respectivamente, mas o que se pode observar é uma menor frequência nas predominâncias desses dois principais setores. 
Figura 4 - Distribuição das direções e horários predominantes do vento para os períodos seco de 2015 e 2016 às $9 \mathrm{~h}$ A e B, $12 \mathrm{~h}$ C e D e 15 h E e F na Floresta Nacional de Caxiuanã, Pará, Brasil.
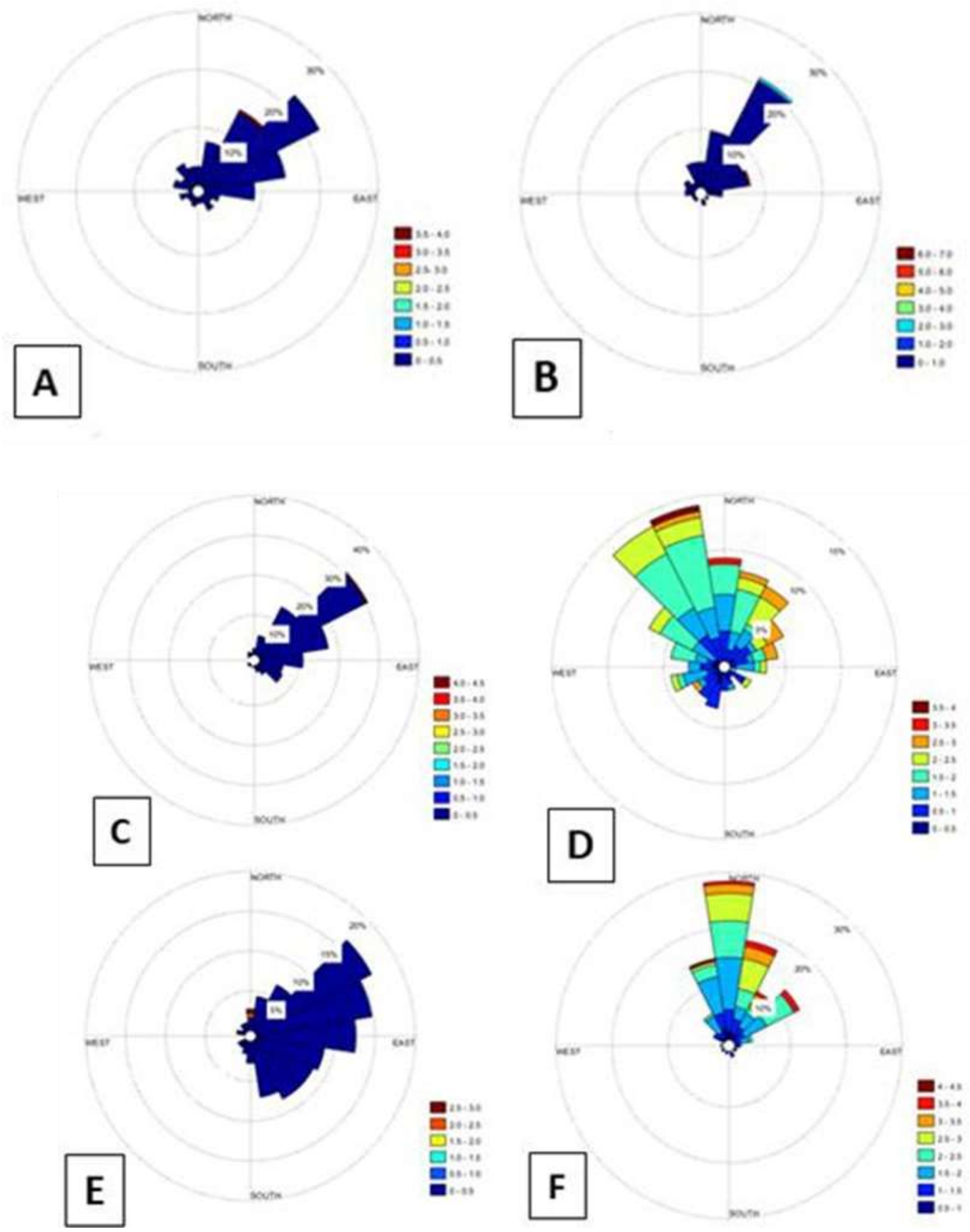

Fonte: Autores.

Tais resultados estão de acordo com os dados da Secretaria de Estado de meio Ambiente e Sustentabilidade do Estado do Pará - SEMAS, a qual aponta a direção oficial dos ventos no estado como sendo de nordeste e sudeste.

Quanto à classificação, nota-se claramente uma maior frequência em relação a ventos fracos para os anos de $2015 \mathrm{e}$ 2016, com 54,57 e 56,50\% respectivamente (Tabela 4). Tapajós et al (2016) realizando medidas a 64 m de altura no km 67 na Flona Tapajós obtiveram um intervalo de classe variando de 2 a 3 m.s-1, com uma frequência de 52,7\%. 
Tabela 4 - Frequência de observação das velocidades do vento (2015 e 2016) acima do dossel na Floresta Nacional de Caxiuanã, Pará, Brasil. Teste de Tukey de comparação de médias (diárias) entre os anos, com 95\% de probabilidade.

\begin{tabular}{l|l|l|l}
\hline & Intervalo & 2015 & 2016 \\
\hline \multirow{2}{*}{ Fraco } & $\geq 2 \mathrm{~m} \cdot \mathrm{s}^{-1}$ & $54.57 \% \mathrm{a}$ & $56.50 \% \mathrm{a}$ \\
\hline \multirow{2}{*}{ Foderado } & $2 \mathrm{a} 4 \mathrm{~m} \cdot \mathrm{s}^{-1}$ & $31.25 \% \mathrm{a}$ & $35.09 \% \mathrm{a}$ \\
\hline & $>4 \mathrm{~m} \cdot \mathrm{s}^{-1}$ & $0.81 \% \mathrm{a}$ & $0.90 \% \mathrm{a}$ \\
\cline { 2 - 4 } & Máxima & & $8.05 \mathrm{~m} \cdot \mathrm{s}^{-1}$ \\
\cline { 2 - 4 } & Média & $6.3 \mathrm{~m} \cdot \mathrm{s}^{-1}$ & $1.86 \mathrm{~m} \cdot \mathrm{s}^{-1}$ \\
\cline { 2 - 4 } & Mínima & $0.11 \mathrm{~m} \cdot \mathrm{s}^{-1}$ & $0.11 \mathrm{~m} \cdot \mathrm{s}^{-1}$ \\
\hline
\end{tabular}

Fonte: Autores.

Quanto à distribuição anual, percebeu-se que o predomínio dos ventos oriundos de NE e L (que somados representam 45,45\% da captação dos ventos Alísios) para 2015, é de 65,97\% para 2016 (Tabela 5).

O restante das frequências observadas 54,55 e 34,03\%, estão distribuídas nos demais setores de direção, sugerindo que circulações locais possam estar influenciando no escoamento do ecossistema estudado, como preconizado por Santana et al. (2016).

Tabela 5 - Distribuição anual das direções predominantes do vento por setores para 2015 e 2016 na Floresta Nacional de Caxiuanã, Pará, Brasil.

\begin{tabular}{l|l|l|l}
\hline \multicolumn{2}{l|}{ Direção vento 2015 } & \multicolumn{2}{l}{ Direção vento 2016 } \\
\hline $\mathbf{N}$ & $13.49 \%$ & N & $10.24 \%$ \\
\hline $\mathbf{L E}$ & $\mathbf{3 4 . 9 1 \%}$ & $\mathbf{L E}$ & $\mathbf{5 4 . 0 2 \%}$ \\
\hline $\mathbf{L}$ & $\mathbf{1 0 . 5 4 \%}$ & $\mathbf{L}$ & $\mathbf{1 1 . 9 5 \%}$ \\
\hline $\mathrm{S}$ & $4.93 \%$ & $\mathrm{~S}$ & $7.30 \%$ \\
\hline SE & $2.56 \%$ & SE & $1.09 \%$ \\
\hline SO & $3.97 \%$ & SO & $1.44 \%$ \\
\hline $\mathrm{O}$ & $2.80 \%$ & O & $1.00 \%$ \\
\hline NO & $13.76 \%$ & NO & $4.94 \%$ \\
\hline
\end{tabular}

Fonte: Autores.

Para ambos os anos, pôde-se observar uma clara diferença sazonal no comportamento do vento para o sítio avaliado. Observa-se que para ambos os períodos do dia os ventos predominantemente foram de NE e L, compreendendo a uma variação angular de $15^{\circ}$ a $105^{\circ}$ de azimute, tanto para o ano de 2015 quanto a 2016. Nunes et al (2010) encontraram direções de NE e SE, sendo a primeira predominante durante o período chuvoso e a segunda para o período seco, neste mesmo sítio experimental, os quais sugerem que a predominância pode estar associada às correntes de ar descendentes associadas às atividades convectivas e as brisas fluviais.

Quando comparada, as médias não apresentaram diferenças estatísticas dentre e entre anos, os valores aqui levantados mostraram-se iguais estatisticamente $(\mathrm{p}$ valor $=0,642$ ) e direções predominantemente de SE e NE. A interação do vento com as florestas causa mudanças no regime de vento local, originando com isso, situações cujo estudo se mostra bastante complexo dado o caráter turbulento e tridimensional na maior parte dos casos (Santos 2013).

\section{Conclusão}

A direção do vento predominantemente foi de NE e L durante os anos avaliados, estando em concordância com a maioria dos estudos situados nesta região do globo em detrimento a confluência dos ventos Alísios, embora estando com sua 
intensidade reduzida em relação a anos ditos normais.

A intensidade dos ventos não apresentou diferença estatística $(p>0,05)$ quando comparada por sazonalidade $\mathrm{e}$ interanual no período observado, e apresentando poucas variações em relação à direção.

Conhecer a estrutura do escoamento acima de formações vegetais, principalmente as florestais, complexas por sinal, é necessário para uma melhor compreensão dos impactos que as mesmas causam no campo de vento.

Os resultados aqui obtidos são de muito interesse no aperfeiçoamento de parametrizações dos processos de troca entre a superfície e a atmosfera, tendo grande aplicação nos estudos de escoamento acima da região tropical, conduzindo à elaboração de melhores prognósticos do tempo, com aplicações na previsão do tempo, degradação ambiental, poluição, agricultura e saúde.

\section{Agradecimentos}

Ao Programa LBA, pelo fornecimento dos dados para realização deste trabalho e a equipe de campo de micrometeorologia da Embrapa Amazônia.

A CAPES pela concessão de bolsa.

Ao Programa de Pós-graduação em Ciências Florestais.

\section{Referências}

Abrão, M. S. (2005). Ventos: Tipos de vento, massas de ar, frentes frias e brisa marítima. http://educacao.uol.com.br.

Alves, E. D. L., \& Silva, S. T. (2011). Direção e velocidade do vento em uma floresta de transição Amazônia-Cerrado no Norte de Mato Grosso, Brasil. Cuiabá: Universidade Federal de Mato Grosso, Boletim Goiano de Geografia, 31(1), 64 - 74.

André, J. C., \& Mahrt, L. (1982). The nocturnal surface inversion and influence of clear-air radiative cooling. Journal of the Atmospheric Sciences, 39(4), 864878 .

Ayoade, J. O. (2007). Introdução à climatologia para os trópicos. (12a ed,), Bertrand Brasil.

Barnard, P. L., Hoover, D., Hubbard, D. M., Snyder, A., Ludka,B. C., Allan, J., Kaminskyg. M., Ruggiero, P., Gallien, T. W., Gabel, L., Mccandless, D., Weiner, H. M., Cohn, Nicholas., Anderson, D. L., \& Serafin, K. A. (2016). Extreme oceanographic force and coastal response due to the 2015- 2016. El Niño. Nature communications. 10.1038/ncomms14365.

Bueno, R. C., Carvalho, L. G., Vianello, R. L., \& Marques, J. J. G. S. M. (2011). Estudo de rajadas de ventos e direções predominantes em Lavras, Minas Gerais, por meio da distribuição gama. Ciência Agrotécnicnologia, 35(4), 789-796.

Cobra-Pará. (2007). Caxiuanã observations in the biosphere, river and atmosphere of Pará.

Cohen, J. C. P., Sá, L. D. A., Nogueira, D. S., \& Gandu, A. W. (2006). Jatos de baixos níveis acima da floresta amazônica em Caxiuanã. Revista Brasileira de Meteorologia, 21(3b), $271-282$.

De Paula, R. R. C. (2007). Investigação numérica do escoamento dentro e acima do dossel de florestas. 2007.160f. Tese Doutorado em Engenharia Mecânica - Pontifícia Universidade Católica do Rio de Janeiro.

Hastenhrath, S. (1985). Climate and circulation of the Tropics. D. Riedel, Dordrecht.

Mohmad, U. (2010). Factors that affect the direction and wind speed. http://www.helium.com.

Mahrt, L., Lee, X., Black, A., Neumann, H., \& Staebler, R. M. (2000). Nocturnal mixing in a forest subcanopy. Agricultural and Forest Meteorology, 101(1), $67-78$

Nogueira, D. S. (2008). Aspectos Observacionais e Numéricos da Interação Floresta- Atmosfera na Amazônia Oriental: Fenômenos Turbulentos Noturnos. 2008.143f.Dissertação Mestrado - Universidade Federal do Pará, Museu Paraense Emílio Goeldi, Empresa Brasisleira de Pesquisa Agropecuária, Belém.

Melo, A. B. C., Rocha, F., \& Sismanoglu, R. A. (2015). Boletim de informações climáticas do CPTEC/INPE, ano 22(08).

Nunes, H. G. G. C., Barreto, P. N., Sousa, A. M. L., Ferreira, R. C., Santos, S. R. Q., Rodrigues, R. S., \& Silva, R. B. C. (2010). Análise da variabilidade média mensal de variáveis meteorológicas na floresta nacional de Caxiuanã/PA de 2000-2004. In: XVI Congresso Brasileiro De Meteorologia, 2010, Belém/PA. Anais. Belém.

Oliveira, P. J., \& Fisch, G. (2001). Perfil de vento em áreas de floresta e pastagem na Amazônia. Acta Amazônica, 31(4), 581-595. 
Research, Society and Development, v. 11, n. 1, e47711122584, 2022

(CC BY 4.0) | ISSN 2525-3409 | DOI: http://dx.doi.org/10.33448/rsd-v11i1.22584

Santana, R. A. S., Do Vale, R. S., Tóta, J., Dos, R. M. N., Fitlzjarrald, D. R., Picanço, G. A. S., Batalha, S. S. A., Gomes, A. C. S, Costa, G. B., Tapajós, R., \& Silva, R. (2016). Características média do vento acima e abaixo do dossel da floresta durante o GO Amazon em um sítio experimental na Amazônia. Ciência e Natura, 38, 152 - 156.

Santos, A. B. (2013). Dinâmica de escoamento do ar acima e dentro de uma floresta tropical densa sobre terreno complexo na Amazônia: aspectos observacionais e de modelagem. 2013. 48f. Dissertação Mestrado em Clima e Ambiente - Instituto Nacional de Pesquisas da Amazônia e Universisade do Estado do Amazonas, Manaus.

Santos, M. J., M. A. F. Silva Dias., \& Freitas, E. D. (2014). Influence of local circulations on wind, moisture, and precipitation close to Manaus City, Amazon Region, Brazil. Journal of Geophysical Research: Atmospheres, 119(13), 233-13,249.

Silva, L. M. (2008). Caracterização de regimes de umidade em regiões tropicais: comparação entre floresta e savana. 2008. 135 f. Dissertação Mestrado em Ciências Ambientais - Universidade Federal do Pará, Belém.

Souza Filho, J. D. C., Ribeiro, A., Costa, M. H., \& Cohen, J. C. P. (2005). Mecanismos de controle da variação sazonal da transpiração de uma floresta tropical no nordeste da Amazônia. Acta Amazônica, 35(2), 223-229.

Strong, C., Fuentes, J. D., Garstang, M., \& Betts, A. K. (2005). Daytime cycle of low-level clouds and the tropical convective boundary layer in southwestern Amazonia. Journal of Applied Meteorology, 44(10), 1607-1619.

Tapajós, R., Da Silva, R., Machado, W., Santana, R., Do Vale, R., \& Ferreira-Júnior, M. (2016). Análise das características do vento sobre a Floresta nacional do Tapajós, Pará, Brasil. Ciência e Natura, 38, 205-208.

Varejão-Silva, M. A. (2006). Meteorologia e Climatologia. Recife.Versão Digital. 443 p.

Vendramini, E. Z. (1986). Distribuições probabilísticas de velocidades do vento para avaliação do potencial energético eólico. 1986. $110 \mathrm{f}$. Tese Doutorado em Agronomia- Faculdade de Ciências Agronômicas, Universidade Estadual Paulista, Unesp, Botucatu. 